\title{
Anti-aggregatory effect of boswellic acid in high-fat fed rats: involvement of redox and inflammatory cascades
}

Mona Kamal Tawfik

Department of Pharmacology, Faculty of Medicine, Suez Canal University, Ismailia, Egypt

Submitted: 17 October 2014

Accepted: 30 October 2014

Arch Med Sci 2016; 12, 6: 1354-1361

DOI: 10.5114/aoms.2016.60675

Copyright $\odot 2016$ Termedia \& Banach

\author{
Corresponding author: \\ Mona Kamal Tawfik PhD \\ Department of Pharmacology \\ Faculty of Medicine \\ Suez Canal University \\ 002-064 Ismailia, Egypt \\ Phone: 002-01222719473 \\ E-mail: dmon_kamal@yahoo. \\ com
}

\begin{abstract}
Introduction: A high-fat diet is one of the main dietary factors promoting platelet aggregation. The present study was conducted to elucidate the involvement of boswellic acid (BA) on the platelet hyperaggregability in HFDfed rats. As platelet hyperaggregability in HFD rats is closely linked to inflammation and enhanced free radical production, the present study was extended to evaluate the anti-inflammatory and anti-oxidative effect of BA on HFD-promoted platelet aggregation.

Material and methods: Rats were assigned to normal, HFD-fed, aspirin-treated $(30 \mathrm{mg} / \mathrm{kg}$ ), and BA-treated (250 and $500 \mathrm{mg} / \mathrm{kg}$ ) groups.

Results: Boswellic acid administration in a high dose was effective in attenuating the severity of hyperlipidemia and platelet aggregation, indicated by lower collagen/epinephrine-induced platelet aggregation, as evidenced by the significant increase $(p<0.05)$ in the circulating platelet count and reduction in the number of thrombi in the lungs. Moreover, it attenuated the oxidative stress and the intensity of inflammatory mediators associated with platelet hyperaggregability, as evidenced by the inhibitory effects on interlukin-1 $\beta$, COX-2 and tumor necrosis factor- $\alpha$, indicating that the antiplatelet activity of BA is likely a consequence of controlling oxidative stress and inflammation.

Conclusions: The present data suggest that BA shows a promising anti-aggregatory effect by attenuating the enhanced hyperlipidemia, oxidative stress and inflammation associated with HFD.
\end{abstract}

Key words: boswellic acid, high-fat diet, inflammation, oxidative stress, platelet aggregation, rat.

\section{Introduction}

Platelets are essential for normal homeostasis. However, excessive activation of platelets that leads to their aggregation plays a major role in the pathogenesis of cardiovascular disorders such as atherosclerosis, cardiac dysfunction and peripheral artery disease [1]. Metabolic abnormalities as a consequence of a high-polyunsaturated-fat diet (HFD) cause platelet hyperaggregability involving enhanced intraplatelet reactive oxygen species (ROS) production and decreased nitric oxide (NO) bioavailability [2]. The relationship between platelet aggregation and inflammation has been previously discussed [3]. Several studies have shown that animals fed high-fat diets are more likely to develop dyslipidemia [4], insulin resistance [5], hepatic steatosis [6], platelet aggregation [7], inflammation [8] and oxidative stress [9]. 
Anti-platelet targets are increasingly used not only for anti-thrombotic prophylaxis, particularly in patients who have had a myocardial infarction or angina pectoris, but also in healthy individuals at increased risk of developing cardiovascular diseases $[10,11]$. However, the major limitation to the longterm use of these targets is the increased risk of intracranial hemorrhage and gastrointestinal bleeding [11]. Given the importance of platelet activation in the pathogenesis of cardiovascular diseases, research is oriented towards the discovery of new, naturally occurring anti-platelet medicinal agents with fewer or no adverse effects that can serve as a useful adjuvant to various anti-platelet targets.

Triterpenoids are known for their anti-oxidant and anti-inflammatory properties. Among thousands of triterpenoids found in plants, boswellic acid (BA) is very promising due to its effectiveness and stability [12, 13]. Boswellic acid is extracted from the gum resin of Boswellia serrata, exhibits a variety of profound effects such as being anti-inflammatory, antioxidant, cancer drug sensitizing, cardio-protective, insulin resistance lowering, and gastro-protective [13-16].

Considering that platelet hyperaggregability in HFD rats is closely linked to inflammation and enhanced free radical production, together with the fact that BA is known for its antihyperlipidemic, antioxidant and anti-inflammatory properties, this compound was a logical candidate for study as its administration could reverse this scenario. So, the aim of this study is to investigate the role of BA in the platelet hyperaggregability in HFD-fed rats.

\section{Material and methods}

\section{Drugs and chemicals}

Boswellic acid was purchased from Advance Physician Formulas Inc. (California, USA) in tablet form; it was dissolved in distilled water. Acetyl salicylic acid was kindly provided by Medical Union Pharmaceuticals (MUP, Ismailia, Egypt). Bovine collagen and epinephrine were purchased from Sigma-Aldrich (St. Louis, MO, USA). Cholesterol was purchased from GFS chemicals (Texas, USA) and bile salts were purchased from SAS Chemicals Co. (Mumbai, India). All the used solvents were of analytical grade and were supplied by Al-Nasr Company for Chemical Industries (Cairo, Egypt).

\section{Animals}

The experiment was performed using 80 healthy male Wistar albino rats with a weight of $170 \pm 30$ g purchased from the Egyptian Organization for Biological Products and Vaccines. Rats were housed in clean, well-ventilated stainless steel cages, maintained on a normal light-dark cycle and temperature $25 \pm 3^{\circ} \mathrm{C}$ throughout the experiment. Standard rodent chow and water ad libitum were provided to the rats and they were left 1 week for acclimatization. All experimental procedures were approved by the institutional animal care and use committee at the Suez Canal University, following the National Institutes of Health guide for the care and use of laboratory animals.

\section{Atherogenic diet}

The high-fat diet (HFD) was composed of $87.7 \%$ standard diet (w/w), 10\% pork fat (w/w), $2 \%$ cholesterol $(\mathrm{w} / \mathrm{w})$ and $0.3 \%$ bile salts $(\mathrm{w} / \mathrm{w})$ [17].

\section{Experimental design}

The experiment was divided into two parts as follows:

\section{Experiment I}

Forty male albino rats were randomly allocated to five groups, eight rats in each. Group I: the normal group: rats fed with normal palatable diet. All the other groups were fed with the HFD for 12 weeks followed by a 4-week therapeutic period. Group II: the HFD-fed control rats. Group III: aspirin-treated (30 mg/kg) group [18]. Group IV: BA-treated $(250 \mathrm{mg} / \mathrm{kg})$ group. Group V: BA-treated $(500 \mathrm{mg} / \mathrm{kg}$ ) group [19]. In general, aspirin and BA were dissolved in distilled water and administered orally using a gastric tube. Experiment I was conducted to obtain data about all the measured parameters and assay with exception of the collagen-induced platelet consumption assay. At the end of the experiment, rats were anesthetized using thiopental sodium (50 mg/kg) and sacrificed by decapitation. Fresh blood samples were collected for the following assays.

Platelet aggregation assay: Blood was collected into a $3.8 \%$ sodium citrate solution (9:1 $\mathrm{V} / \mathrm{V})$. Then, samples were centrifuged immediately at $160 \times \mathrm{g}$ for $15 \mathrm{~min}$ at room temperature to prepare platelet-rich plasma (PRP). After that, PRP was transferred into plastic tubes and the remaining blood was centrifuged at $3000 \times \mathrm{g}$ for $10 \mathrm{~min}$ to obtain the platelet-poor-plasma (PPP). Platelet count in PRP was adjusted to $5 \times 10^{8} / \mathrm{ml}$ using PPP. Platelet aggregation was performed after addition of $5 \mu \mathrm{g} / \mathrm{ml}$ collagen (Chrono-Log corp.) using a dual channel aggregometer (Clot 2, SEAC- Radim Company, Italy). Results were expressed as a percentage of aggregation; the extent of aggregation was estimated by the change in light transmission [20].

Blood collection and serum separation: Fresh blood samples (1 $\mathrm{ml}$ of blood) were collected in a dry centrifuge tube and were allowed to stand for 30 min before centrifugation at $3000 \times \mathrm{g}$ for $15 \mathrm{~min}$. Then, sera were separated, collected in 
clean tubes and stored at $-80^{\circ} \mathrm{C}$ until used for the following assays.

Determination of serum lipid profile: Serum total cholesterol, triglycerides (TGs), low-density lipoproteins (LDL) and high-density lipoproteins $(\mathrm{HDL})$ were determined using commercial kits, purchased from Bio Diagnostics (Cairo, Egypt). These parameters were determined enzymatically according to the manufacturer's protocol using an ultraviolet-visible spectrophotometer (UV-1601PC, Shimadzu, Kyoto, Japan).

Determination of malondialdehyde (MDA) and reduced glutathione (GSH): Tissues malondialdehyde (MDA) was estimated according to the spectrophotometric method of Ohkawa et al. [21] using 1,1,3,3-tetramethoxypropane as a standard. Concentration of reduced glutathione (GSH) was measured spectrophotometrically using commercial kits according to the instructions of the manufacturer [22].

Determination of superoxide dismutase (SOD) and catalase (CATA): The activity of SOD was assessed as described by Marklund [23], and CATA activity was measured according to Aebi [24].

Determination of serum level of IL-1 $\beta$, COX-2 and TNF- $\alpha$ : Enzyme-linked immunosorbent assay (ELISA) kits for IL-1 $\beta$, COX-2 and TNF- $\alpha$ were obtained from Glory Science Co., Ltd (Del Rio, TX, USA) and were used to measure these parameters in serum samples. Assays were performed according to the manufacturer's instructions using an automated ELISA reader (Metertech, M960).

\section{Experiment II}

In a separate set of experiments, another set of rats (40 rats) - identical in the study groups - were used to develop a rat model of pulmonary thrombo-embolism for thrombogenesis assay. Using these rats, a platelet count was obtained before and af-

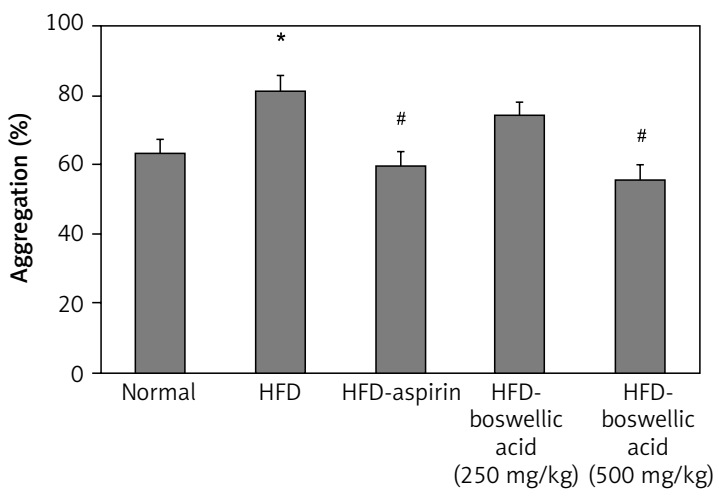

Figure 1. Platelet aggregation assay in the experimental groups. HFD: high-fat diet. Data are presented as mean \pm SEM and analyzed using oneway ANOVA followed by Tukey's post-hoc test at $p<0.05(n=8)$

${ }^{\star}$ Compared to normal group. "Compared to HFD group. ter injection of a collagen/epinephrine mixture, and lung specimens were taken for histopathological examination as detailed in the next sections.

Collagen-induced platelet consumption: Collagen was given to induce platelet activation to perform a pulmonary thrombo-embolism model as described previously by Seth et al. [25] with minor modifications. A mixture of bovine collagen $(1000 \mu \mathrm{g} / \mathrm{kg})$ plus epinephrine $(200 \mu \mathrm{g} / \mathrm{kg})$ was injected into the rat tail vein.

Platelet count: Platelet count was carried out immediately before and $3 \mathrm{~min}$ after injection of the collagen/epinephrine mixture. Blood samples were collected and anticoagulated with a $10 \%$ EDTA solution. After mixing, platelets were counted automatically on a Cell-Dyn 1700 instrument (Abbott Laboratories, USA).

Histopathological examination of the lungs: After blood collection, rats were anesthetized with thiopental sodium ( $50 \mathrm{mg} / \mathrm{kg}$ ) and killed by decapitation. Then, the chest was opened and the lungs were dissected and fixed in a $10 \%$ phosphate-buffered paraformaldehyde solution. Tissues were dehydrated and embedded in paraffin and sectioned at 4- $\mu \mathrm{m}$ and stained with hematoxylin and eosin $(H+E)$. The lung specimens were then examined blindly under a light microscope. The number of thrombi per microscopic field was counted as described by Decrem et al. [26] with slight modification. The thrombi were counted at $10 \times$ magnification; data are presented as the mean for 10 fields for each rat.

\section{Statistical analysis}

All data were tabulated and expressed as mean \pm SEM and analyzed employing the Statistical Package for the Social Sciences (SPSS, version 17, Chicago, IL, USA). Comparisons among groups were carried out using one-way ANOVA. The difference of mean values between groups was assessed by Tukey's post-hoc test. All $p$-values reported are two-tailed and a $p$-value $<0.05$ was considered to be significant.

\section{Results}

\section{Effect of aspirin or BA on HFD-induced platelet aggregation}

Figure 1 shows that the percentage of platelet aggregation was significantly higher $(p<0.05)$ in the high-fat diet fed rats compared to the normal control group. Additionally, rats fed with the HFD exhibited higher collagen/epinephrine-induced platelet aggregation, as evidenced by the reduction $(p<0.05)$ in the circulating platelet count (Figure 2 B) and an increase in number of thrombi in the lungs in comparison with those fed with a normal palatable diet ( $p<0.05$, Figure 2 C). 
A

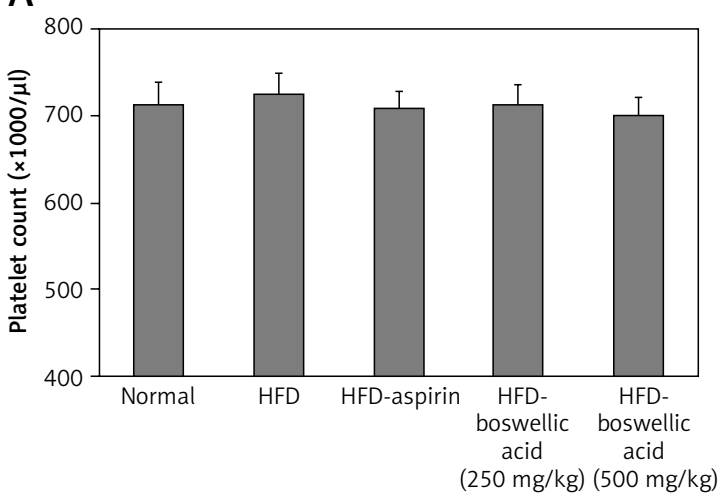

C

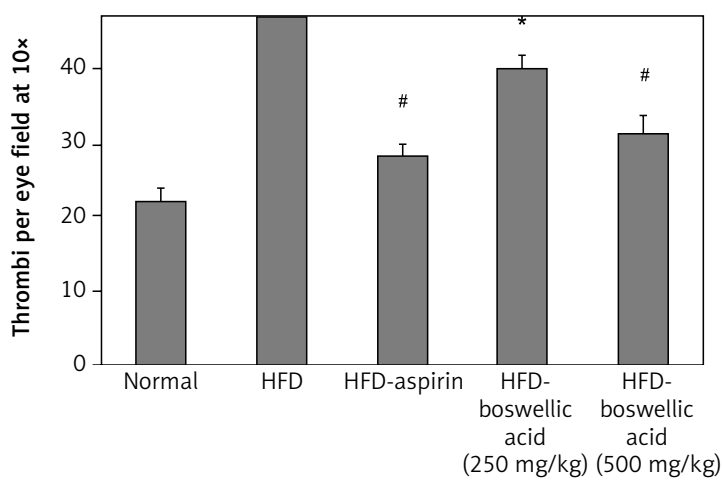

B

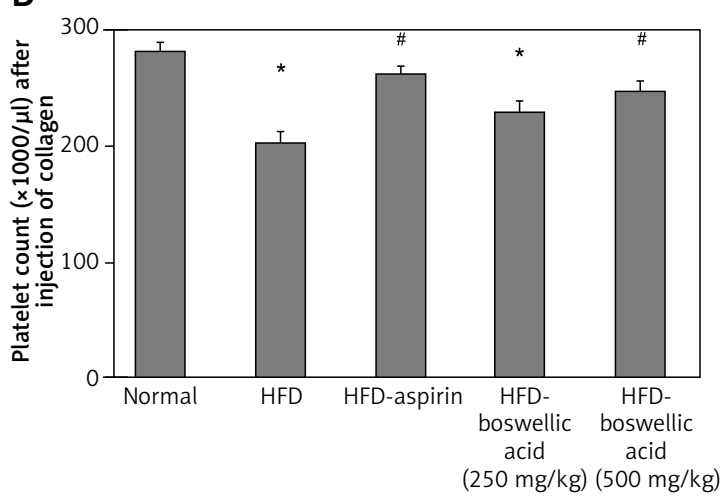

Figure 2. Effect of boswellic acid or aspirin on circulating platelets. A - Platelet count before i.v. injection of collagen $(1000 \mu \mathrm{g} / \mathrm{kg})$ plus epinephrine $(200 \mu \mathrm{g} / \mathrm{kg})$. B - Platelet count 3 min after i.v. injection of collagen-epinephrine mixture. $\mathbf{C}$ - Number of thrombi in the lungs of rats 3 min after injection of collagen epinephrine mixture

HFD - high-fat diet. Data are presented as mean \pm SEM and analyzed using one-way ANOVA followed by Tukey's post-hoc test at $p<0.05(n=8)$. *Compared to normal group. "Compared to HFD group.

Boswellic acid in a high dose was effective in attenuating the severity of HFD-induced platelet aggregation in comparison with the HFD control group ( $p<0.05$, Figure 1 ). Moreover, BA in a high dose attenuated collagen/epinephrine-induced platelet aggregation, as evidenced by the increase $(p<0.05)$ in the circulating platelet count (Figure $2 \mathrm{~B}$ ) and a decrease in the number of thrombi in the lungs in comparison with the high-fat fed rats $(p<0.05$, Figure $2 \mathrm{C})$.

\section{Effect of aspirin or BA on HFD-induced oxidative stress}

In the current study, a high-fat diet induced oxidative stress in the form of a significant increase of MDA concentrations associated with a significant reduction in GSH, SOD activities, and CATA levels in comparison with the normal control group $(p<$ 0.05 , Figure 3$)$. These deleterious effects associated with HFD were improved $(p<0.05)$ by treatment with either aspirin or BA in comparison with the HFD control group. Notably, BA in a high dose induced a significant improvement in oxidative stress markers compared to aspirin ( $p<0.05$, Figure 3 ).

\section{Effect of aspirin or BA on HFD-induced increase in inflammatory mediators}

Figure 4 shows that the HFD increased ( $p<$ $0.05)$ production of the inflammatory mediators
IL-1 $\beta$, COX- 2 and TNF- $\alpha$ compared to the normal control group. These elevations in inflammatory mediators associated with HFD were $(p<0.05$, Figure 4) ameliorated by treatment with either aspirin or BA, as evidenced by the inhibitory effects on interlukin-1 $\beta$, COX-2 and TNF- $\alpha$.

\section{Effect of aspirin or BA on HFD-induced hyperlipidemia}

Table I shows that high-fat fed rats exhibited hyperlipidemia, as is evidenced by the significant $(p<0.05)$ elevation in serum total cholesterol, TG and LDL levels and decline in HDL level. The BA in a high dose was effective in attenuating the severity of hyperlipidemia in comparison with the HFD control group ( $p<0.05$, Table I). Notably, the aspirin-treated group exhibited a non-significant improvement in the severity of hyperlipidemia compared to the HFD control group ( $p>0.05$, Table I).

\section{Discussion}

Given the importance of platelet activation in the pathogenesis of cardiovascular diseases, research is oriented towards the discovery of antiplatelet agents with improved efficacy for preventing or treating arterial or venous thrombosis [27]. Although a number of anti-platelet targets have been identified to address this issue including COX-1, the P2Y12 receptor, the integrin $\alpha$ llb $\beta 3$, 
A

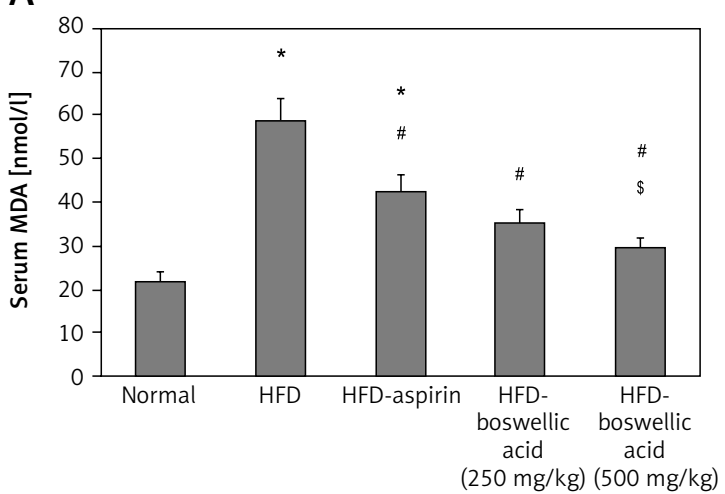

C

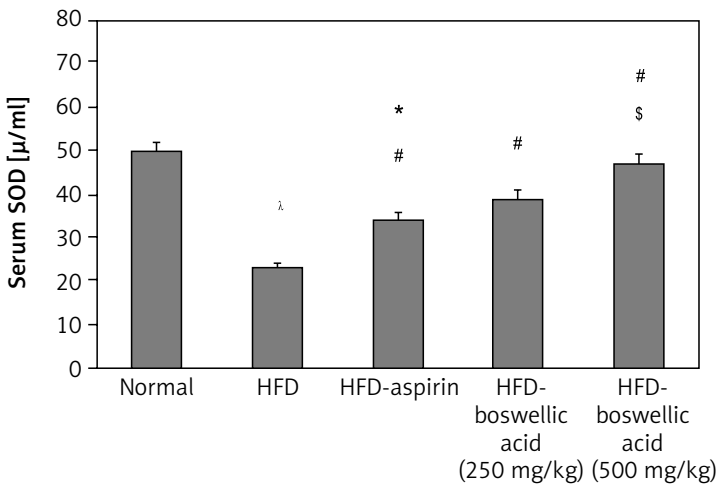

B

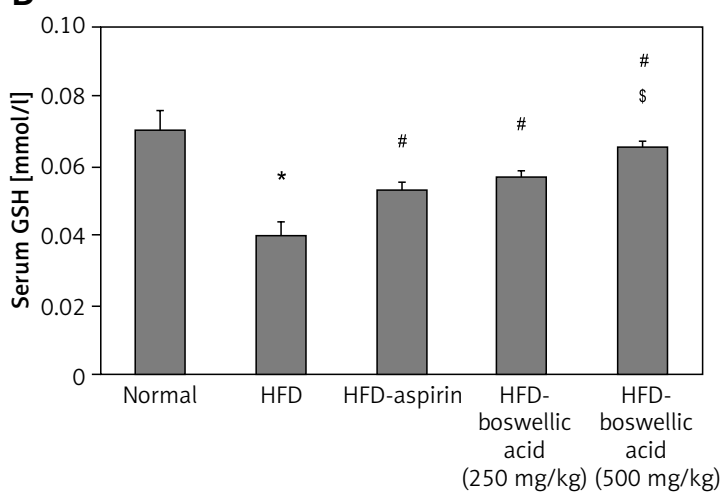

D

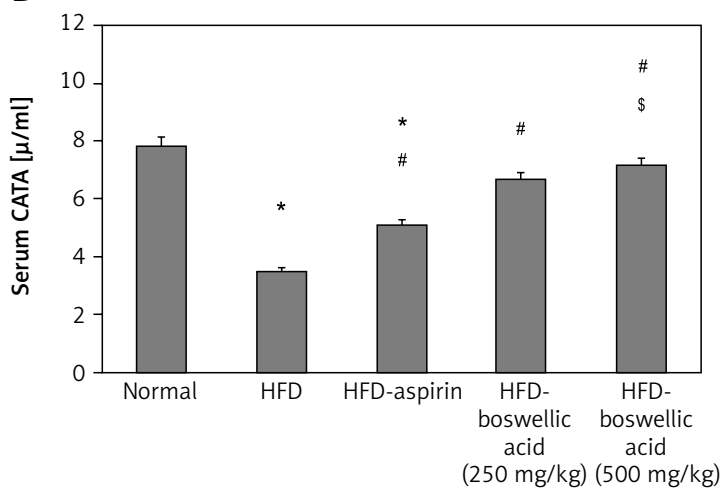

Figure 3. Serum level of MDA (A), GSH (B), SOD (C), CATA (D) in the experimental groups

HFD - high-fat diet. Data are presented as mean \pm SEM and analyzed using one-way ANOVA followed by Tukey's post-hoc test at $p<0.05(n=8)$. ${ }^{*}$ Compared to normal group. ${ }^{*}$ Compared to HFD group. ${ }^{5}$ Compared to aspirin.

A

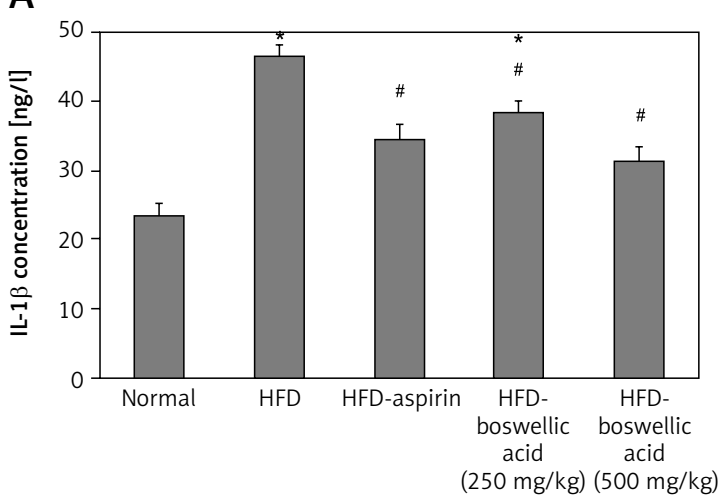

C

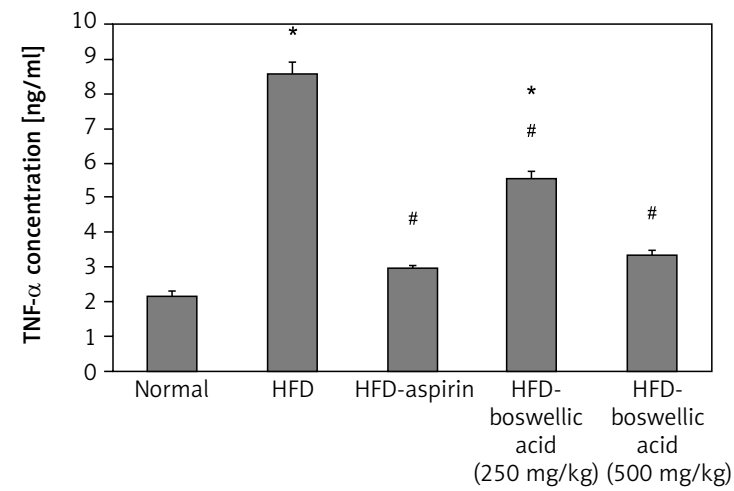

B

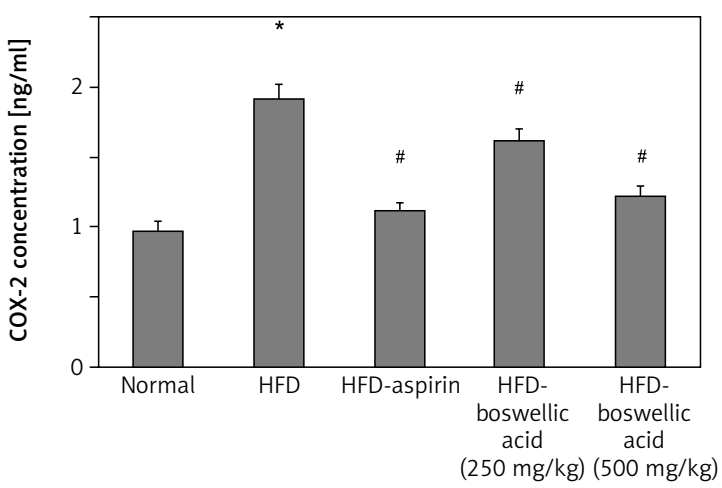

Figure 4. Serum level of interlukin-1 $\beta$ (A), COX-2 (B) and TNF- $\alpha$ (C) in the experimental groups HFD - high-fat diet. Data are presented as mean \pm SEM and analyzed using one-way ANOVA followed by Tukey's post-hoc test at $p<0.05(n=8)$. *Compared to normal group. "Compared to HFD group. 
Table I. Effect of treatment with boswellic acid (250 or $500 \mathrm{mg} / \mathrm{kg}$ ) on serum total cholesterol, triglycerides, HDL-C and LDL-C in hyperlipidemic rats

\begin{tabular}{|c|c|c|c|c|}
\hline Groups & $\begin{array}{c}\text { Total cholesterol } \\
{[\mathrm{mg} / \mathrm{dl}]}\end{array}$ & $\begin{array}{l}\text { Triglycerides } \\
\text { [mg/dl] }\end{array}$ & $\begin{array}{c}\mathrm{HDL}-\mathrm{C} \\
{[\mathrm{mg} / \mathrm{dl}]}\end{array}$ & $\begin{array}{l}\text { LDL-C } \\
{[\mathrm{mg} / \mathrm{dl}]}\end{array}$ \\
\hline Normal & $45.2 \pm 4.2$ & $27.73 \pm 1.8$ & $32.2 \pm 2.4$ & $15.3 \pm 1.2$ \\
\hline HFD & $79.3 \pm 6.6^{*}$ & $59.43 \pm 5.2^{*}$ & $15.5 \pm 1.8^{*}$ & $39.4 \pm 3.9^{*}$ \\
\hline HFD + aspirin & $71.3 \pm 26.8^{*}$ & $55.32 \pm 4.3^{*}$ & $19.2 \pm 17.6^{*}$ & $31.2 \pm 4.2^{*}$ \\
\hline HFD + boswellic acid $(250 \mathrm{mg} / \mathrm{kg})$ & $69.1 \pm 21.4^{*}$ & $53.3 \pm 1.9^{*}$ & $20.12 \pm 19.2^{\star}$ & $33.9 \pm 4.8^{*}$ \\
\hline HFD + boswellic acid $(500 \mathrm{mg} / \mathrm{kg})$ & $52.0 \pm 20.4^{\# \$}$ & $37.0 \pm 2.6^{\# 5}$ & $28.12 \pm 21.6^{\#}$ & $26.22 \pm 5.7^{\#}$ \\
\hline
\end{tabular}

Rats were fed with a HFD for 12 weeks. HFD - high-fat diet. Data are expressed as mean \pm S.E.M. and analyzed using one-way ANOVA followed by Tukey's post-hoc test at $p<0.05$. ${ }^{*}$ Compared to normal group at $p<0.05$, "\#compared to HFD group, ${ }^{5}$ compared to aspirin. $n=8$.

and more recently the protease-activated receptor- 1 , these targets often result in a significant increase in risk of bleeding, which may lead to pathologies as serious as the thrombosis they were meant to treat, including intracranial hemorrhage and gastrointestinal bleeding [11, 12]. The interest in finding naturally occurring components with anti-inflammatory and antioxidant properties to serve as a useful anti-aggregator adjuvant to various anti-platelet targets is increasing [13]. One group of such compound is triterpenoids. Among thousands of triterpenoids found in plants, BA is very promising due to its effectiveness and stability [28].

As a high-fat diet is one of the main dietary factors promoting platelet aggregation and involved in the pathogenesis and development of cardiovascular thrombotic complications, the present study was conducted to investigate the involvement of BA in the platelet hyperaggregability in HFD rats. As platelet hyperaggregability in HFD rats is closely linked to inflammation [3] and enhanced ROS production [7], our study was extended to evaluate the anti-inflammatory and anti-oxidative effect of BA on HFD-promoted platelet aggregation. Consistent with many lines of evidence [2, 29-33] that emphasized that platelet aggregation is fully activated and accelerated by a high-fat cholesterol diet, the present results revealed that rats fed a high-fat cholesterol diet exhibited higher collagen/epinephrine-induced platelet aggregation, as evidenced by the decrease in circulating platelet count and the increase in number of thrombi in the lungs. The platelet hyperaggregability during HFD may in part be related to hyperlipidemia, as evident from the elevated serum total cholesterol, TG and LDL levels and the decline in HDL level. Wang et al. [30] and Ito et al. [32] emphasized that platelet sensitivity and aggregation are enhanced with hyperlipidemia.

Another explanation could be attributed to the presence of enhanced ROS production. Trocha et al. [34] emphasized that conditions including dyslipidemia, obesity, and diabetes mellitus are associated with aggravated oxidative stress. Compatible with findings from previous studies [2, 7 , 31] linking platelet hyperaggregability in HFD rats with enhanced oxidative stress, a rise in MDA levels and depletion in the antioxidant enzyme pool were observed, as evident from the declined activity of GSH, SOD, and CATA.

As inflammation plays a crucial and modulating role in various diseases [35], the relationship between platelet aggregation and acute inflammation has been previously discussed $[3,8]$. In line with this hypothesis, we observed that platelet hyperaggregability was associated with increased production of the inflammatory mediators IL-1 $\beta$, COX-2 and TNF- $\alpha$. Ziccardi et al. [36] and Damas et al. [37] emphasized that the pro-inflammatory and pro-oxidant state that accompanies the HFD has been suggested to be involved in endothelial dysfunction and plaque destabilization, providing a connection between inflammation, oxidative stress and platelet hyperaggregability in metabolic abnormalities as a consequence of $\operatorname{HFD}[2,38]$.

The results of the present study also revealed that BA was effective in attenuating hyperlipidemia-promoted platelet aggregation indicated by lower collagen/epinephrine-induced platelet aggregation, as evidenced by the increase in the circulating platelet count and the reduction in the number of thrombi in the lungs. Consistent with findings from previous studies [39, 40], the present results clearly demonstrated an improvement in lipid profile associated with BA administration. Another explanation could be afforded by Ali and Mansour [41], who emphasized that platelet hyperaggregability associated with HFD was prevented by the use of an antioxidant, indicating the critical role for intracellular ROS in this phenomenon. Similarly to the elsewhere published data [42-44], the results of the present study revealed that antioxidants, by altering the oxidant/antioxidant balance, attenuated HFD-induced hyperaggregability in rats.

As increased production of inflammatory mediators results in endothelium dysfunction as well 
as alterations in the coagulation system [2, 45], the interest in finding naturally occurring components with potent anti-inflammatory properties is increasing. One potential treatment to reduce inflammatory mediators involves the use of BA, which has shown efficacy as an anti-inflammatory agent [15]. The current results are compatible with those reporting the inhibitory role of $\mathrm{BA}$ on serum level of IL- $1 \beta[43,44,46]$, COX-2 and TNF- $\alpha$ $[44,47,48]$. It is obvious that the anti-platelet activity of BA is most likely a consequence of COX-2, IL-1 $\beta$ and TNF- $\alpha$ inhibition. The results of this study demonstrated that BA administration in a high dose, by its known anti-oxidant and antiinflammatory properties [14], exerted an attenuating effect on the extent and severity of ROS production and inflammation, indicating that the anti-platelet activity of BA is likely a consequence of controlling oxidative stress and inflammation.

In conclusion, the present data suggest that BA shows a promising anti-aggregatory effect by attenuating the enhanced hyperlipidemia, oxidative stress and inflammation associated with HFD. Consequently its administration may warrant further attention as a potential adjuvant to existing medications that are used to treat patients with platelet hyperaggregability.

\section{Conflict of interest}

The author declares no conflict of interest.

\section{References}

1. Haouari M, Jardin I, Mekhfi H, et al. Urtica dioica extract reduces platelet hyperaggregability in type 2 diabetes mellitus by inhibition of oxidant production, $\mathrm{Ca} 2+$ mobilization and protein tyrosine phosphorylation. J Appl Biomed 2007; 5: 105-13.

2. Monteiro P, Morganti R, Delbin M, et al. Platelet hyperaggregability in high-fat fed rats: a role for intraplatelet reactive-oxygen species production. Cardiovasc Diabetol 2012; 11: 11-5.

3. Prescott S, Mcintyre T, Zimmerman G, Stafforini D. Lecture in thrombosis: molecular events in acute inflammation. Arterioscler Thromb Vasc Biol 2002; 22: 727-33.

4. Li W, Shi Y, Yang R, et al. Effect of somatostatin analog on high-fat diet-induced metabolic syndrome: involvement of reactive oxygen species. Peptides 2010; 31 625-9.

5. Samane S, Christon R, Dombrowski L, et al. Fish oil and argan oil intake differently modulate insulin resistance and glucose intolerance in a rat model of dietaryinduced obesity. Metab Clin Exp 2009; 58: 909-19.

6. Kajikawa S, Harada T, Kawashima A, Imada K, Mizuguchi K. Highly purified eicosapentaenoic acid prevents the progression of hepatic steatosis by repressing monounsaturated fatty acid synthesis in high-fat/high sucrose diet-fed mice. Prostagland Leukotr Essent Fatty Acids 2009; 80: 229-38.

7. Anfossi G, Russo I, Trovati M. Platelet dysfunction in central obesity. Nutr Metab Cardiovasc Dis 2009; 19: 440-9.
8. Coy-Barrera E, Cuca-Suarez L. In vitro anti-inflammatory effects of naturally-occurring compounds from two Lauraceae plants. Ann of the Brazi Acad Sci 2011; 83: 1397-402.

9. Hee H, Seon L. Effects of Artemisia capillaris ethyl acetate fraction on oxidative stress and antioxidant enzyme in high-fat diet induced obese mice. Chem Biol Interact 2009; 179: 88-93.

10. Hankey G. Antiplatelet therapy for the prevention of recurrent stroke and other serious vascular events: a review of the clinical trial data and guidelines. Curr Med Res Opin 2007; 23: 1453-62.

11. Yeung J, Holinstat M. 12-Lipoxygenase: a potential target for novel anti-platelet therapeutics. Cardiovasc Hematol Agents Med Chem 2011; 9: 154-64.

12. Cicero A, Reggi A, Parini A, Borghi C. Application of polyunsaturated fatty acids in internal medicine: beyond the established cardiovascular effects. Arch Med Sci 2012; 8: 784-93.

13. Ammon $\mathrm{H}$. Boswellic acids in chronic inflammatory dis eases. Planta Med 2006; 72: 1100-16.

14. Poeckel D, Werz O. Boswellic acids: biological actions and molecular targets. Curr Med Chem 2006; 13: 3359-69.

15. Anthoni C, Laukoetter M, Rijcken E, et al. Mechanisms underlying the anti-inflammatory actions of boswellic acid derivatives in experimental colitis. Am J Physiol Gastrointest Liver Physiol 2006; 29: 1131-7.

16. Singh S, Khajuria A, Taneja S, et al. The gastric ulcer protective effect of boswellic acids, a leukotriene inhibitor from Boswellia serrata, in rats. Phytomedicine 2008; 15 : 408-15.

17. Pan $\mathrm{M}$, Song $\mathrm{Y}, \mathrm{Xu}$ J, Gan H. Melatonin ameliorates nonalcoholic fatty liver induced by high-fat diet in rats. J Pineal Res 2006; 41: 79-84.

18. Sun W, Gerhardinger C, Dagher Z, Hoehn T, Lorenzi M. Aspirin at low-intermediate concentrations protects retinal vessels in experimental diabetic retinopathy through non-platelet-mediated effects. Diabetes 2005; 54: 3418-26.

19. Elshazly S, Abd El Motteleb D, Nassar N. The selective 5-LOX inhibitor 11-keto-beta-boswellic acid protects against myocardial ischemia reperfusion injury in rats: involvement of redox and inflammatory cascades. Naunyn-Schmiedeberg's Arch Pharmacol 2013; 386: 823-33.

20. Piccione G, Grasso F, Fazio F, Giudice E. The effect of physical exercise on the daily rhythm of platelet aggregation and body temperature in horses. Vet J 2008; 176: 216-20.

21. Ohkawa H, Ohishi N, Yagi K. Assay for lipid peroxides in animal tissues by thiobarbituric acid reaction. Anal Biochem 1979; 95: 351-8.

22. Griffith O. Determination of glutathione and glutathione disulfide using glutathione reductase and 2-vinylpyridine. Anal Biochem 1980; 106: 207-12.

23. Marklund S. Regulation by cytokines of extracellular superoxide dismutase and other superoxide dismutase isoenzymes in fibroblasts. J Biol Chem 1992; 267: 96102.

24. Aebi H. Catalase in vitro. Meth Enzymol 1984; 105 : 121-6.

25. Seth P, Kumari R, Dikshit M. Alterations in the free radical generation and nitric oxide release from rat peripheral polymorphonuclear leukocytes following thrombosis. Thromb Res 1997; 87: 279-88.

26. Decrem Y, Rath G, Blasioli V, et al. Ir-CPI, a coagulation contact phase inhibitor from the tick Ixodesricinus, in- 
hibits thrombus formation without impairing homeostasis. J Exp Med 2009; 206: 2381-95.

27. Agrawal S, Saraswati S, Mathur R, Pandey M. Antitumor properties of boswellic acid against ehrlich ascites cells bearing mouse. Food Chem Toxicol 2011; 49: 1924-34.

28. Liu J, Duan R. LY294002 enhances boswellic acid-induced apoptosis in colon cancer cells. Anticancer Res 2009; 29: 2987-92.

29. Waqar M, Mahmood Y. Anti-platelet, anti-hypercholesterolemic and anti-oxidant effects of ethanolic extracts of brassica oleracea in high fat diet provided rats. World Appl Sci 2010; 8: 107-12.

30. Wang W, Zhang H, Gao G, Bai Q, Li R, Wang X. Adiponectin inhibits hyperlipidemia-induced platelet aggregation via attenuating oxidative/nitrative stress. Physiol Res 2011; 60: 347-54

31. Haimeur A, Ulmann L, Mimouni V, et al. The role of Odontella aurita, a marine diatom rich in EPA, as a dietary supplement in dyslipidemia, platelet function and oxidative stress in high-fat fed rats. Lipids Health Dis 2012; 11: 147-60

32. Ito H, Uehara K, Matsumoto $\mathrm{Y}$, et al. Cilostazol inhibits accumulation of triglyceride in aorta and platelet aggregation in cholesterol-fed rabbits. PLoS One 2012; 7: e39374.

33. Wang Y, Wang J, Guo L, Gao X. Antiplatelet effects of qishen yiqi dropping pill in platelets aggregation in hyperlipidemic rabbits. Evid-Based Complement. Alternat Med 2012; 12: 1-5.

34. Trocha M, Merwid-Ląd A, Chlebda E, et al. Influence of ezetimibe on selected parameters of oxidative stress in rat liver subjected to ischemia/reperfusion. Arch Med Sci 2014; 10: 817-24.

35. Ciebiada M, Kasztalska K, Gorska-Ciebiada M, Barylski M, Gorski P. Expression of IL-7 receptor in human peripheral regulatory T cells. Arch Med Sci 2013; 9: 555-60.

36. Ziccardi P, Nappo F, Giugliano G, et al. Reduction of inflammatory cytokine concentrations and improvement of endothelial functions in obese women after weight loss over one year. Circulation 2002; 105: 804-9.

37. Damas J, Vaehre T, Yndestad A, et al. Interleukin-7-mediated inflammation in unstable angina: possible role of chemokines and platelets. Circulation 2003; 107: 2670-6.

38. Ertek S, Cicero A. Impact of physical activity on inflammation: effects on cardiovascular disease risk and other inflammatory conditions. Arch Med Sci 2012; 8: 794804.

39. Alemika T, Ojerinde S. Brine shrimp toxicity of acidic fractions of boswellia dalzielii gum resin. J Pharm Biores 2005; 2: 137-40.

40. Pandey R, Singh B, Yamini B. Tripathi, extract of gum resins of Boswellia serrata $L$. inhibits lipopolysaccheriode induced nitric oxide production in rat macrophages along with hypolipidemic property. Ind J Exp Biol 2005; 43: 509-16.

41. Ali E, Mansour S. Boswellic acids extract attenuates pulmonary fibrosis induced by bleomycin and oxidative stress from gamma irradiation in rats. Chin Med 2011; 30: 6-36.

42. Saraswati S, Agrawal S. Antiangiogenic and cytotoxic activity of boswellic acid on breast cancer MCF-7 cells. Biomed Prevent Nutr 2012; 2: 31-7.

43. Bansal N, Mehan S, Kalra S, Khanna D. Boswellia serrata-frankincense (A Jesus Gifted Herb); an updated pharmacological profile. Pharmacologia 2013; 4: 457-63.
44. Zhang Y, Ning Z, Lu C, et al. Triterpenoid resinous metabolites from the genus boswellia: pharmacological activities and potential species-identifying properties. Chem Cent I 2013; 7: 153-69.

45. Won S, Tang X, Suh S, Yenari M, Swanson R. Hyperglycemia promotes tissue plasminogen activator-induced hemorrhage by increasing superoxide production. Ann Neurol 2011; 70: 583-90.

46. Gayathri B, Manjula N, Vinaykumar K, Lakshmi B, Balakrishnan A. Pure compound from boswellia serrata extract exhibits anti-inflammatory property in human PBMCs and mouse macrophages through inhibition of TNFalpha, IL-1beta, NO and MAP kinases. Int Immunopharmacol 2007; 7: 473-82.

47. Cao H, Yu R, Choi Y, et al. Discovery of cyclooxygenase inhibitors from medicinal plants used to treat inflammation. Pharmacol Res 2010; 61: 519-24.

48. Park B, Prasad S, Yadav V, Sung B, Aggarwal B. Boswellic acid suppresses growth and metastasis of human pancreatic tumors in an orthotopic nude mouse model through modulation of multiple targets. PLoS One 2011; 6: e26943. 